\title{
The Basic Slippery Slope Argument
}

\author{
Douglas WaLton
}

Centre for Research in Reasoning, Argumentation and Rhetoric

Department of Philosophy

University of Windsor

Windsor, $O N$

Canada N9B 3P4

dwalton@uwindsor.ca

\begin{abstract}
Although studies have yielded a detailed taxonomy of types of slippery slope arguments, they have failed to identify a basic argumentation scheme that applies to all. Therefore, there is no way of telling whether a given argument is a slippery slope argument or not. This paper solves the problem by providing a basic argumentation scheme. The scheme is shown to fit a clear and easily comprehensible example of a slippery slope argument that strongly appears to be reasonable, something that has also been lacking.
\end{abstract}

Résumé: Bien que des études aient donné une taxonomie détaillée des types d'arguments de pente glissante, ils n'ont pas réussi à identifier un schème d'argumentation de base qui s'applique à tous. Par conséquent, il n'y a aucun moyen de savoir si un argument donné est un argument de la pente glissante ou non. Cet article permet de résoudre ce problème par un schème d'argumentation de base qui correspond à un exemple clair et facile à comprendre d'un argument de pente glissante qui semble fortement raisonnable, quelque chose d'absent dans les études sur ces arguments.

Keywords: vagueness, compressed slippery slope arguments, argumentation schemes, genetics, eugenics, ethical argumentation, critical questioning

\section{Introduction}

The slippery slope argument has become a prominent and controversial form of reasoning in biomedical ethics (van der Burg, 1991; Holtug, 1993; Launis, 2002; Saliger, 2007) and legal argumentation (Schauer, 1985; Volokh, 2002; Rizzo and Whitman, 2003). Notably since (Beardsley, 1966), it has been treated as a distinctive type of argument in its own right in the logic textbooks, typically under the heading of informal fallacies. However, it remains a central problem that although different definitions of the slippery slope argument as a distinctive type of argument have been put forward in the literature, they don't agree with each other, and don't go far enough to provide a pre-

(C) Douglas Walton. Informal Logic, Vol. 35, No. 3 (2015), pp. 273-311. 
cise model of the structure of the argument that is adequate to move forward to solve the problem of how to analyze and evaluate slippery slope arguments. Corner et al. $(2011,134)$ noted that while "it is simple to produce an intuitive characterization of slippery slope arguments, they [these characterizations of slippery slope arguments] have resisted attempts to provide a comprehensive definition." Is it this apparent intuitive simplicity of slippery slope arguments that in fact makes them difficult to define in a way that is clear, precise and comprehensive? The definitions given in the literature identify some characteristics of the slippery slope argument well enough, but do not include enough characteristics to show precisely how they are combined to make any of them workable as a comprehensive definition.

There has been considerable work done on analyzing many interesting and controversial cases where the slippery slope argument has been used. This work has enabled the development of a typology providing argumentation schemes for the analysis and evaluation of different kinds of slippery slope arguments. Corner et al. $(2011,134)$ noted that the definition of the slippery slope argument in Walton (1992) distinguished four types of slippery slope arguments, depending on the mechanism that moves the argument forward: those based on causal mechanisms, those that set precedents, those that are attributable to the vagueness of concepts, and a fourth type that combines features from each of the three other types. Corner et al. $(2011,134)$ note however that although the Walton (1992) analysis provides a detailed taxonomy, it fails to identify the core features common to all slippery slope arguments, and therefore does not provide a central definition that applies to all slippery slope arguments. Lode $(1999,1492)$ has even gone so far as to claim that there is no single, distinctive form of the slippery slope argument.

This paper provides an argumentation scheme for a basic slippery slope argument that models the structure of this type of argument and enables the construction of a definition that represents its core features. The slippery slope as a type of argument is defined by stating ten requirements that need to be made for a given argument to fit this category, and showing how the basic scheme is related to its four subtypes and other closely related types of arguments.

\section{Sources of the slippery slope argument}

Alfred Sidgwick is the earliest known author of a book on informal logic, at least to my knowledge, to identify, illustrate and

(C) Douglas Walton. Informal Logic, Vol. 35, No. 3 (2015), pp. 273-311. 
define the commonly used type of argument corresponding to what is nowadays called the slippery slope argument. However, Sidgwick $(1910,40)$ called it the thin edge of the wedge objection, defining it as follows.

We must not do this or that, it is often said, because if we did we should be logically bound to do something else which is plainly absurd or wrong. If we once begin to take a certain course there is no knowing where we shall be able to stop within any show of consistency; there would be no reason for stopping anywhere in particular, and we should be led on, step by step into action or opinions that we all agree to call undesirable or untrue.

Later informal logic textbooks, notably including Beardsley $(1966,176)$ took the direction of including the slippery slope argument under the general heading of informal fallacies. However, although Beardsley described slippery slope arguments as often alarming enough to be persuasive, he added the comment (Beardsley, $4^{\text {th }}$ ed., 1975, 150) that slippery slopes can be good arguments in some instances. Currently the standard treatment of the slippery slope argument in the logic textbooks is to include it under the section on fallacies, but also to occasionally concede that such arguments are not always fallacious. ${ }^{1}$ But of course, evaluating whether such an argument is fallacious depends on how the type of argument is defined.

\section{Structure of the slippery slope argument}

Rizzo and Whitman (2003) cited three characteristics they claim are common to all slippery slope arguments: (1) an initial, seemingly acceptable decision, (2) a dangerous outcome that is unacceptable, and (3) a process or mechanism leading from the initial decision to the dangerous outcome. These three characteristics surely are common to all slippery slope arguments, but until we know more about the nature of the mechanism that leads from the initial decision to the dangerous outcome, and how it works

\footnotetext{
${ }^{1}$ A Google Ngram search for the expression 'slippery slope fallacy' on August 12, 2014 found that there were zero occurrences in 1960, and although the number went slightly upward by 1980 , there was a tenfold increase peaking in the 1990s. The search was not restricted to informal logic textbooks, but covered all occurrences of the expression in all books in the Google database at that time.
}

(C) Douglas Walton. Informal Logic, Vol. 35, No. 3 (2015), pp. 273-311. 
to drive the argumentation forward from the start point to the end point, we have not comprehensively defined the slippery slope type of argument. One problem is that it does not provide a way of distinguishing between slippery slope arguments and arguments from negative consequences, a much more common form of argumentation.

Corner et al. $(2011,135)$ offer a better definition that states that slippery slope arguments have four components: (1) an initial proposal for action, (2) an undesirable outcome, (3) a belief that allowing the action will lead to a reevaluation of the undesirable outcome in the future, and (4) the rejection of the initial action proposed, based on this belief. This definition is more comprehensive, because it brings out a fourth characteristic, but it still fails to give a precise and sufficiently detailed account of the mechanism through which allowing the initial action leads to some sequence of actions or procedure that drives the sequence forward from the start point to the end point. This fourth characteristic is emphasized in the definition offered by Volokh (2002, 1030): "I think the most useful definition of a slippery slope is one that covers all situations where decision $A$, which you might find appealing, ends up materially increasing the probability that others will bring about decision $B$, which you oppose." However, this definition is even more minimal than the previous two.

It is a problem cited by Walton (1992) that when students first learn about slippery slope arguments, they tend to label any argument from negative consequences as a slippery slope argument. This kind of labeling or (mislabeling) can have the effect of shifting the burden of proof against the arguer who appears to have committed a fallacy, and is therefore obliged to somehow try to respond. Volokh $(2002,1030)$ has noted this reaction.

When someone says "I oppose partial-birth abortion bans because they might lead to broader abortion restrictions," or "I oppose gun registration because it might lead to gun prohibition," the common reaction is "That's a slippery slope argument."

A problem with this reaction is that in the absence of a precise definition of the slippery slope argument, it is unclear whether the two arguments cited are merely argument from negative consequences. But the mere labeling of the argument as a slippery slope may put the other side on the defensive.

Corner et al. (2011, 135) do distinguish between slippery slope arguments and arguments from negative consequences. They call argument from negative consequences "general con- 
sequentialist arguments," citing the example of opposing the legalization of cannabis because it would lead to an increase in chronic obstructive lung disease. In contrast they cited an example of what they consider a genuine slippery slope argument: if cannabis were legalized, attitudes towards harder drugs might become more positive, and in the future heroin might also become legalized. According to Saliger (2007, 342), "slippery slope arguments belong to the class of practical consequence arguments," agreeing with the view of Corner et al. (2011) that there is a distinction to be drawn between the two types of arguments, one being a subset or special subtype of the other. On Saliger's view, (2007, 343), not all result arguments (arguments from consequences) are slippery slope arguments. Drawing this distinction is very helpful to working towards an adequate definition of the slippery slope argument, but by itself, none of these definitions provides a precise enough or fully adequate comprehensive account of the slippery slope argument to explain how the mechanism from the start point to the end point works, and what its requirements are as a distinctive type of argument.

Holtug (1993, 403) defines the slippery slope argument as having the following kind of structure as an argument with three premises and conclusion.

Premise 1: An agent is considering whether or not to bring about an action or class of actions $A$.

Premise 2: The argument is brought forward that if $A$ is carried out, another action $B$ will inevitably or probably follow.

Premise $3: B$ is morally undesirable

Conclusion: The agent ought to refrain from bringing about $A$.

He adds "Normally, $B$ is not thought to follow directly from $A$, but through a series of steps $A_{1}-A_{n}$." This definition is better but still not fully adequate, because the mechanisms of how the slope is slippery and how the sequence is driven forward by loss of control are not accounted for.

\section{Relation to vagueness and argument from negative consequences}

As shown in section 3, there has been a difficulty in clearly distinguishing between the slippery slope type of argument and other kinds of arguments it is closely related to. This difficulty

(C) Douglas Walton. Informal Logic, Vol. 35, No. 3 (2015), pp. 273-311. 
has been compounded by mixing the slippery slope argument with another type of argument recognized in some of the texts called the argument of the beard (Walton, 1996). The argument of the beard is a criticism used by one party to attack an opponent by alleging that the opponent's argument is fallacious because the opponent used a vague term falling on a continuum. The first of the nine textbooks that included a discussion of this fallacy between 1930 and 1967 was that of Thouless (1930). Thouless $(1930,183)$ described it as the fallacy of arguing that there is no difference between two things because there is a continuum between them that exhibits no sharp dividing line. Damer $(1980,37)$ described it as the fallacy of arguing that it is impossible or arbitrary to make a definite distinction between two things on a continuum because there is no precise point on the continuum where a distinction can be drawn between the one thing and the other. This kind of argument is at least in some cases fallacious because, as we now know, natural language argumentation, such as the kind of argumentation that takes place in legal contexts, is open-textured. This means that a given term, even if precisely defined, will always be open to vagueness and reinterpretation when applied to a new case it has not yet been tested against. Vagueness is a common phenomenon in the using of a verbal criterion to draw classifications that are required in argumentation. But vagueness is not always incorrect or fallacious in arguments, and is not always eliminable (Walton, 1996, 256).

The argument of the beard is very closely related to the sorites type of argument widely known to the ancient Greek philosophers. A paradox called the heap (sorites) has been attributed to Eubulides, a student of Euclides, an older contemporary of Plato. The paradox of the heap can be posed in the form of an argument with two premises leading to an absurd conclusion (Kneale and Kneale, 1962, 114).

Premise 1: If you take one grain away from a heap, it makes no significant difference-you still have a heap.

Premise 2: Each time you repeat this step, it makes no difference, because one grain is too small to make a difference between something being a heap or not.

Conclusion: Repeated long enough, the conclusion of this reasoning will become absurd, for it will become obvious that what is left can no longer be described as a heap. 
This form of argument is different from the kind of slippery slope argument we are familiar with in the logic textbooks because it is not about a sequence of actions that leads to some dangerous outcome where a warning is being offered not to take the first step in the sequence. However, we can see that there are some similarities. Both forms of argument are about a sequence. In both cases, once the sequence moves forward and is not stopped, the result is a problematic outcome.

A comparable example of the sorites argument was called the bald man paradox, described by the following sequence of questions and replies (Kneale and Kneale, 1962, 114).

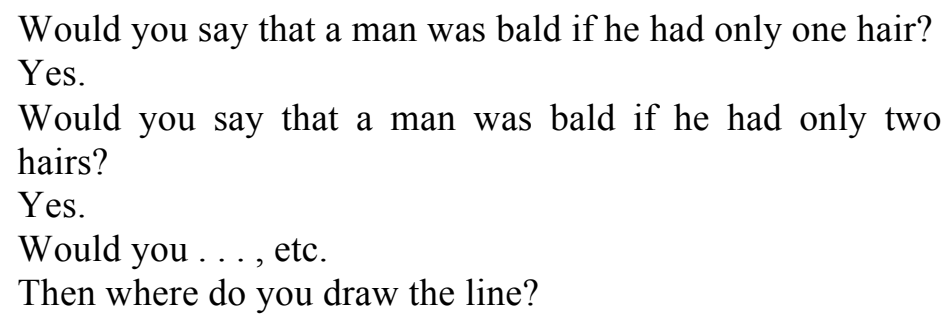

This is a paradox, but putting it in this format shows that it could also be used as the basis for a clever argument to attack someone who is having difficulty drawing the line, or making a clearcut line of demarcation on a continuum.

Cicero (Academica 93, H. Rackham, tr., Loeb Library, 1951, 586) understood that such paradoxes arise from vagueness because there is no clear cutoff point. If we are asked by gradual stages whether a person is a rich man or a poor man we do not know at what point in the addition or subtraction to give a definite answer. In such cases, since there is no way of setting absolute limits, a series of questions asked in gradual stages can be used to press ahead. So for example, 1,000,000 grains of sand is a heap of sand, and a heap of sand minus one grain is still a heap, but repeated actions of removing one grain at a time eventually forces you to accept the conclusion that one grain of sand is a heap. Using such a clever argument to drive an opponent to an absurd conclusion is a sophistical strategy that could perhaps be associated with fallacies.

As we will see, once we have looked at a clear example of the slippery slope argument and analyzed its components and structure in detail in section 7, it will become evident how vagueness is an essential component in the slippery slope argument. But at the same time it will become evident that vagueness is not the whole story of the slippery slope argument. There are two factors that are combined to make a slippery slope ar- 
gument slippery, as will be shown in section 8 . The first is the connection between a so-called gray area caused by indeterminacy, typically arising from vagueness, on a continuum in a contemplated sequence of actions. The second factor is loss of control combined with this indeterminacy. Once the agent contemplating taking the first step in the sequence proceeds along the sequence and enters the indeterminate gray area, it loses control over its capability to stop moving ahead. The ultimate outcome of the combination of these two factors is held by the slippery slope arguer to result in the series of steps in the sequence leading to a catastrophic outcome.

Once the structure of the slippery slope argument has been more clearly and precisely defined by the argumentation scheme proposed in section 7, the distinction between it and the argument from negative consequences will become more evident. All slippery slope arguments are instances of argument from negative consequences, but they are special instances that have the feature of the sequence of actions leading into and out of the gray zone. To make the distinction between the two types of arguments more precise for those who have to identify instances of each of them in texts of natural language discourse, the characteristics of argument from negative consequences have to be defined more precisely.

Argument from negative consequences cites the consequences of a proposed course of action as a reason against taking that course of action. This type of argument also has a positive form, in which positive consequences of an action are cited as a reason for carrying it out. The following are the two basic argumentation schemes for arguments from consequences (Walton, Reed and Macagno, 2008, 332), where $A$ represents a state that could be brought about by an agent.

The first scheme is called argument from positive consequences.

Premise: If $A$ is brought about, good consequences will plausibly occur.

Conclusion: Therefore $A$ should be brought about.

The other scheme of the pair is called argument from negative consequences.

Premise: If $A$ is brought about, then bad consequences will plausibly occur.

Conclusion: Therefore $A$ should not be brought about.

(C) Douglas Walton. Informal Logic, Vol. 35, No. 3 (2015), pp. 273-311. 
Argumentation from consequences offers a reason to accept a proposal for action tentatively, subject to exceptions that may arise as new circumstances become known. An instance of argument from consequences can be stronger or weaker, depending on its initial plausibility and the critical questions that have been used to attack it.

Nobody would be likely to challenge the view that argument from negative consequences is a legitimate and reasonable form of argument in many instances, even though it can go wrong and is associated with incorrect or fallacious arguments in some instances. We use this kind of argument all the time, for example when we consider whether or not to take a medication and look on the bottle to see its listed side effects.

\section{Compressed slippery slope arguments}

In many examples that are identifiable as slippery slope arguments, the argument is presented in a compressed form that jumps to the conclusion seemingly in one quick step. Consider the following three examples of slippery slope arguments studied by Corner, Hahn and Oaksford $(2011,133)$.

1. If we allow gay marriage, then in the future people will want to marry their pets.

2. If voluntary euthanasia is legalized, then in the future there will be more cases of medical murder.

3. If we accept voluntary ID cards in the UK, we will end up with compulsory ID cards in the future.

Corner, Hahn and Oaksford $(2011,133)$, comment on the first example that few would agree that homosexual marriages are the beginning of the slippery slope to interspecies marriages, but add that this argument was actually put forward by a group called the American Family Research Council in 2004. On the second example they comment that this argument is somewhat more plausible. On the third example they comment that it seems extremely likely that ID cards would become compulsory if they were introduced in the UK. So here we have a range of slippery slope arguments in which some are more plausible than others.

In all three of these cases, it is hard to decide for sure on the basis of the given evidence whether they are really slippery slope arguments or should better be classified as simply arguments from negative consequences. In the case of the third one,

(C) Douglas Walton. Informal Logic, Vol. 35, No. 3 (2015), pp. 273-311. 
it is not even really certain whether it is an argument. On the surface it looks to be merely a prediction in the form of a conditional statement. To reconstruct it as an argument we have to do a lot of filling in and making assumptions with very little textual evidence to go on. It looks like it is a slippery slope argument on the basis that it is claiming that ending up with compulsory ID cards in the future might be perceived to be a very dangerous outcome, and that the first step in some sequence of events leading to this outcome is the acceptance of voluntary ID cards in the UK. Some idea of how to make it into a slippery slope argument is given by Corner, Hahn and Oaksford $(2011,133)$ who make the comment that it is likely that ID cards in the UK would become compulsory if they were introduced if it turns out that they were to function as an effective security measure. This outcome might make them a necessity to guard against security threats. However, it is not altogether certain whether this additional contextual information should be inserted as part of the argument. This uncertainty poses a problem, because we do not want to commit the straw man fallacy by inserting an implicit premise in an argument that the arguer has not actually stated.

A special feature of these three examples is their compressed format. We usually think of slippery slope arguments as built around a connected sequence of actions and consequences starting from an initial action or policy and then proceeding through a sequence to an eventual outcome. However in many examples, the three examples of slippery slope argument given above being cases in point, only the initial action and the eventual outcome are specified.

On February 3, 2015, Britain became the first country to allow the creation of babies with genetic material from three persons. Mitochondria, the powerhouses of our cells generate the energy that our cells need to function properly. About 100 children a year suffer from faults with mitochondria that are often agonizing and fatal. Mitochondria contains DNA, and therefore intervention using germline therapy results in genetic changes that will be inherited by the mother's children, and all their children, and so forth. The slippery slope argument used against this procedure is that it will be "the first step on the road to designer babies" (No author given, Oh Baby, The Economist, February 7, 2015, page 12). The reply made to the slippery slope argument given in the Economist article states: "[This complaint] is as weak as any other slippery slope argument: approving one procedure does not mean automatically approving others." Here we have an instance of a condensed version of the slippery slope 
argument arguing against going ahead with this genetic intervention. And then we have a counterargument claiming that the slippery slope argument is weak because approving one procedure does not mean automatically approving others.

In this case the slippery slope used to attack the argument for genetic intervention does not seem to be inherently unreasonable. The problem is that it is so compressed that we cannot tell what the steps are supposed to be between this first step and the alleged ultimate outcome of "designer babies," taken by many to be a catastrophic outcome because of its association with eugenics. The term 'eugenics' is associated in the public mind with the Nazi euthanasia program and other crimes aided by medical policies in Nazi Germany (Bashford and Levine, 2010).

However the reply made to the slippery slope argument also seems reasonable, because it makes the generally valid point that approving one procedure does not automatically lead to approving others. In other words there it makes the reasonable point that more evidence needs to be given by the proponent showing how the intervening steps between the first step in the catastrophic outcome are of such a nature that somewhere along the line there is a descent into a set of circumstances where it is no longer possible to go back and as a result the catastrophic outcome becomes inevitable.

So in this instance, the argumentation between the two parties is a stalemate, until the proponent replies to the respondent by filling in enough gaps in the slippery slope sequence so that the burden of proof shifts back again to the respondent's side to present further critical questions or counterarguments. The hypothesis suggested by such cases is that in many instances of slippery slope arguments some of the premises of an argument are not stated explicitly, but are tacitly assumed. An enthymematic argument is an argument that only makes sense if one or more of its components (premises or conclusions) are inserted, typically using Gricean implicature (Grice, 1975; Macagno and Walton, 2013). Each case has to be analyzed and the arguments in it classified using the textual evidence of the case. Here the hypothesis is proposed that it is the presence of the second through the sixth premises (in the argumentation scheme presented in section 7) that enables us to determine in any given case whether the argument fits the scheme for the slippery slope argument as well as fitting the more general scheme of argument from negative consequences. 


\section{The drug example}

In this paper it is argued that slippery slope arguments can be reasonable in some instances. Previous authors have maintained this thesis (Govier, 1982; Walton, 1992). But as one looks through the literature on slippery slope arguments, it is difficult or even impossible to find a single example of one that meets all the requirements for being a reasonable argument. Many of the leading examples in the literature on euthanasia, freedom of speech and so forth, are controversial and interesting, but expressed in such a compressed way, and mixed with other arguments on a controversial issue that it is hard to figure out what the premises and the conclusion are supposed to be. Another problem is that the slippery slope is often supposed to be a fallacy, but it seems to be presupposed in the literature that under the right conditions it can be a rational argument. On the other hand, there is no clear example of it being a rational argument in the literature, at least of a kind that can be used as a paradigm example.

The closest example of an argument that looks to be simple and a fairly reasonable instance of the slippery slope argument is the following one quoted from (Burgess, 1993, 169).

Many slippery slope arguments in applied ethics are simple in structure, highly specific in scope and modest in the practical counsel they offer us. A good example involves the question whether to prescribe drug A or drug $\mathrm{B}$ to a patient. For the condition concerned, A might score best on almost all relevant criteria whilst B does adequately on all, if less well on some. Suppose, further, that there is no realistic possibility of switching drugs in mid-treatment. Now, if A leads to tolerance, eventually needing to be administered in a lethal dose, a simple and sound slippery slope argument leads to the conclusion that drug B is to be preferred.

This example compares arguments about the side effects of two drugs. Each of the arguments is clearly an argument from negative consequences. The example is a good one because it is an argument that seems quite reasonable. If it is not possible for the patient to stop taking drug A in mid-treatment, and continuing to take it will lead to the death of the patient at some point past mid-treatment, the argument does seem to fit the slippery slope category. Giving the patient advice not to start taking drug A certainly does seem to be practical counsel given these circum-

(C) Douglas Walton. Informal Logic, Vol. 35, No. 3 (2015), pp. 273-311. 
stances. Unfortunately however, the example is one of those compressed instances of the argument where the sequence of actions leading from the initial step to the eventual outcome is not explicitly stated enough for us to grasp its essential features.

But still, the example has some good features, and it is possible to build on it by making up a hypothetical example about taking drugs where the intervening sequence of actions has been set out more explicitly. This example is easily recognizable as a common sort of argument that appears to be a reasonable, even if defeasible, argument. Let's call it the drug example. This example is set out in a narrative fashion in the following two paragraphs.

Alice is a student. One day one of her fellow students who had been taking heroin at a party offered Alice a free sample, saying it would make her feel very good. Alice was hesitant because her father Bob had warned her never to take drugs because they can be addictive and eventually lead to a situation where they ruin your life entirely. Alice decides to think about the situation before accepting any offer of drugs. After that she told Bob about what happened at the party. Bob repeated his earlier advice to her, but was disturbed about the incident and thought after some reflection that he should try to offer Alice a better argument that would be more convincing to persuade her not to take drugs such as heroin. He did a little research on addiction, and presented a more carefully structured argument to her.

He told her that taking a drug of this kind is characterized by immediate gratification but easily leads to a situation of dependence because as it is taken again and again a dependency is created where the body needs more and more of the drug in order to get the same state of pleasure. This condition of tolerance to the drug leads to withdrawal symptoms, such as intense cravings, nausea, and tremors that can only be relieved by taking increasingly larger amounts of it. At this point it can become extremely difficult or impossible to stop taking drug, even with medical intervention, even though continuing to take it can be catastrophic to health and normal function, even though it may lead to serious medical disability or death. Based on these facts, Bob reiterated that Alice should never take an addictive drug such as heroin even one time because it is addictive, and once you start taking it, even a few times, it becomes progressively harder to stop, and you never know when it becomes so hard to stop that you can't do it. He argued that the long-term effects of such an addiction on the body are ruinous to health and well-being. He said that a sensible person knows that he or she should never 
take such a drug, even once, because the risks are too great. He also added some other arguments, including the argument that taking heroin is illegal, and if anyone finds out you have taken such a drug it can ruin your reputation.

In this case, Bob is giving advice to Alice as she makes a decision about what choice to make when confronted by someone offers her a drug such as heroin. His advice takes the form of an argument, based on what Bob claims are some facts about addictive substances. Bob's argument seems fairly reasonable. Many of us would even say that his argument is very reasonable and that he is giving Alice some good advice.

On the other hand it might look to an audience that Bob's argumentation could be classified as a slippery slope argument. Indeed Alice might reply to it by saying, "That's a slippery slope argument." And since slippery slope arguments have traditionally been treated as fallacious, it might look to the audience, at least initially, that there is something terribly wrong with this argument.

\section{A proposed argumentation scheme for the slippery slope argument}

Strictly speaking, to be a slippery slope, an argument has to meet requirements of its argumentation scheme. The scheme has a characteristic set of premises and a conclusion defined as statement forms that contain variables and constants. The constants have to be defined externally and restrictions are placed on what entities can fit into the variables. There needs to be identification rules that help a coder engaged in argument identification to determine whether a given argument in a natural language text fits a particular scheme or not. There are also sets of basic characteristics defining a particular type of argument such as the slippery slope argument that can help to differentiate between instances of it and other comparable types of arguments.

There are six basic characteristics common to all slippery slope arguments (Walton, 1992, 208). These six characteristics are included in the ten basic characteristics listed below, but have been reformulated in order to make them expressible within the structure of a formal multi-agent deliberation dialogue. There are two agents taking turns making moves in the form of speech acts to each other. One kind of speech act is that of putting forward an argument, another move made as a speech act is that of replying to the argument by criticizing it or making an

(C) Douglas Walton. Informal Logic, Vol. 35, No. 3 (2015), pp. 273-311. 
objection to it. In a deliberation dialogue, the agents taking part in the proceedings are trying to arrive at a decision on what to do in a particular set of circumstances, or perhaps to agree on a policy. The participant contemplating carrying out the particular action in the case of a slippery slope argument will be called the agent. The responding party, who is criticizing the argument or objecting to it by putting forward a slippery slope argument, will be called the critic. For purposes of the ease of exposition, the former agent will always be designated as "she," and the other agent will be designated as "he."

Using this terminology, the ten basic characteristics of the slippery slope type of argument can be formulated as follows.

1. The agent is deliberating on whether to take an action or accept a policy.

2. The critic postulates a sequence of further actions that will move forward as consequences of the agent's carrying out the initial contemplated action.

3. At the beginning, each single step in the sequence appears to be a small or not very significant one, but as the sequence proceeds the consequences tend to become more serious.

4. There are factors that help to propel the argument and series of consequences along the sequence, making it progressively harder for the agent to resist continuing to move ahead.

5. At the beginning of the sequence the agent retains control of whether to stop moving ahead.

6. However during some interval along the sequence of actions, the agent loses control of the possibility of stopping from moving ahead.

7. This interval cannot be precisely specified, meaning that the agent never knows during the earlier steps in the sequence at what precise point her loss of control will take place.

8. Once the agent enters into the interval where she loses control, she cannot go back and must continue the procedure of bringing about further consequences in the sequence.

9. Past the loss of control interval, the sequence inevitably proceeds to an endpoint, an outcome that is catastrophic for the agent, and for the other agents taking part in the deliberation. 
10. The critic argues that the agent should not take the first step, because if she does, she will be led to unpredictably lose control, and then will be unable to avoid the catastrophic outcome.

These ten characteristics are codified in the basic argumentation scheme for the slippery slope argument below, a substantially revised version of the comparable argumentation scheme for the slippery slope argument formulated in (Walton, 1992, 199-200). Let's call it the basic argumentation scheme for the slippery slope argument.

Initial Premise: An agent $\alpha$ is considering carrying out an action $A_{0}$.

Sequential Premise: Carrying out $A_{0}$ would lead to $A_{1}$, which would in turn lead to carrying out $A_{2}$, and so forth, through a sequence $A_{2}, \ldots, A_{x}, \ldots A_{y}, \ldots, A_{n}$.

Indeterminacy Premise: There is a sequence $A_{0}, A_{1}, A_{2}, \ldots$ $, A_{x}, \ldots A_{y}, \ldots, A_{n}$ that contains a subsequence $A_{x}, \ldots$ $A_{y}$ called the gray zone where $x$ and $y$ are indeterminate points.

Control Premise: $\alpha$ has control over whether to stop carrying out the actions in the sequence until $\alpha$ reaches some indeterminate point in the gray zone $A_{x}, \ldots A_{y}$.

Loss of Control Premise: Once $\alpha$ reaches the indeterminate point in the gray zone $A_{x}, \ldots A_{y}, \alpha$ will lose control and will be compelled to keep carrying out actions until she reaches $A_{n}$.

Catastrophic Outcome Premise: $A_{n}$ is a catastrophic outcome that should be avoided if possible.

Conclusion: $A_{0}$ should not be brought about.

The factors referred to in characteristic 4 are called drivers. A driver is a catalyst that helps to propel the argument along the sequence in the argument, making it progressively harder for the agent to resist continuing. There can be a driver such that once the agent takes a given step $A_{i}$ then she will also be naturally, but not initially, inevitably impelled forward to take the next step $A_{j}$ of the sequence. There can be more than one driver in a given slippery slope argument, and different drivers can come into play at different parts of the sequence, as illustrated in the drug example below. Drivers include such factors as precedent, public acceptance, or vagueness, but can include many other factors as well. In the full slippery slope argument previously 
modeled by (Walton, 1992, 199), the driver is a climate of social opinion. To cite an example, public acceptance of voluntary euthanasia may lead to wider public acceptance of the policy of terminating life, which could lead to greater tolerance for policies of ending life other than on a voluntary basis. Or to cite another kind of example of a driver, taking a drug could become habit-forming and eventually lead to an addiction.

To show how Bob's argument in the drug example fits the scheme, it is necessary to identify the premises and the conclusion of Bob's argument. When this argument is fitted to the scheme, there are seven premises, two drivers and one conclusion. Below, each premise is given a name as a label.

Initial Premise: You are considering taking a drug, in this instance heroin.

First Sequential Premise: One thing leads to another, and so it is very likely that you will continue taking the drug, and may suffer no visible harmful effects.

First Driver: Taking a drug such as heroin typically results in immediate gratification that leads to forward to repeating this feeling of pleasure more than once.

Indeterminacy Premise: People vary concerning their susceptibility to a drug such as heroin. Some become addicted very easily while others can control it for a long time.

Control Premise: At first you have control over whether to stop taking the drug at any point.

Loss of Control Premise: Once you have become addicted, you have lost control, and you cannot stop taking the drug.

Second Driver: Once the body has adjusted to the drug, and has become dependent on it, withdrawal symptoms make it very hard to reduce or discontinue taking the drug.

Second Sequential Premise: Once you have lost control, you are impelled forward to the catastrophic outcome, such as losing your family, your friends, your career, your financial assets, your reputation and your health, or even your life. ${ }^{2}$

\footnotetext{
${ }^{2}$ This sequence should not be seen as inevitable. There are exceptions. For example, you are impelled to the final outcome unless you can get medical help. But even if you can get medical help, the addiction may be too strong.
}

(C) Douglas Walton. Informal Logic, Vol. 35, No. 3 (2015), pp. 273-311. 
Catastrophic Outcome Premise: This outcome is catastrophic and should be avoided at all costs.

Conclusion: You should not take this drug.

The problem posed by this example is how to model the drivers. It seems that the drivers should be additional premises, but they are optional, meaning that in some cases no drivers are identified while in other cases one or more drivers can be identified. This example suggests that the drivers can be modeled as additional premises that support particular premises of the kind stated explicitly in the basic argumentation scheme for the slippery slope argument. Following this method, the first driver is taken as a premise that supports the first sequential premise, while the second driver is taken as an additional premise that supports the loss of control premise. This way of modeling the drivers in the example is shown in figure 1. It follows the format of the Carneades Argumentation System (CAS) by showing the ultimate conclusion at the left, and using the lines going from the premises to a circular argument node that goes to the conclusion (Gordon, 2010). A pro argument is represented by a plus symbol in the argument node.

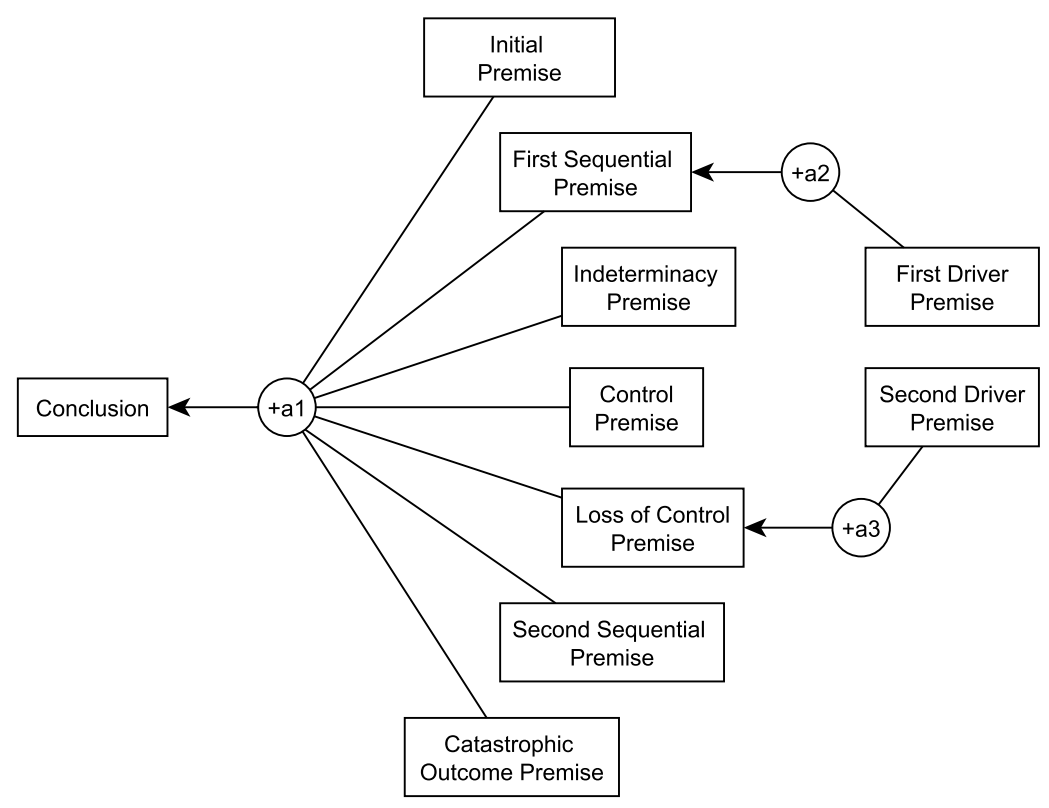

Figure 1: Modeling the Slippery Slope Argument in the Drug Example 
In figure 1 the scheme for the basic slippery slope argument fits the node containing the notation $+a 1$. It is an important requirement for this scheme that the second through sixth premises be present (either explicitly or implicitly). Without these premises, the argument clearly is an instance of argument from negative consequences, but not one that can also be properly classified as a slippery slope argument. In more compressed versions of the slippery slope argument, one or more of the six essential premises are unstated but implicit. In this case we have shown the sequential premise as split into two. This is not an essential feature of slippery slope arguments but it is useful to split them in some cases when showing drivers.

The broader structure of the slippery slope argument represented by this scheme has a modus tollens form comparable to the form of argument known as reductio ad absurdum.

If the initial step $A_{0}$ is taken (as can be seen by applying the next six premises) the catastrophic outcome $A_{n}$ will occur, or at least will be likely to occur in the future.

The catastrophic outcome $A_{n}$ should carefully be avoided. Therefore the initial step $A_{0}$ should not be taken.

It can also be seen that this broader structure of the argument is a special instance of the scheme for argument from negative consequences. These remarks suggest the beginning of a classification system. In this paper, the task of building a classification system showing the connections between these related types of argument is not attempted. But in the literature (Govier, 1982; Walton, 1992), several types of slippery slope arguments have been identified, such as the precedent type, the linguistic type (based on vagueness of a term), and the causal type. Some remarks are needed how these types can be classified as special instances of the basic argumentation scheme formulated above.

A scheme for the causal type of slippery slope is quoted from (Walton, 1992, 93).

Initial Action Premise: $S_{0}$ is up for consideration as a proposal that seems initially like something which should be brought about.

Causal Sequence Premise: Bringing about $S_{0}$ would plausibly cause (in the given circumstances, as far as we know) $S_{1}$, which would in turn plausibly cause $S_{2}$, and so forth, through the sequence $S_{2}, \ldots, S_{n}$ (for some finite $n$ ).

(C) Douglas Walton. Informal Logic, Vol. 35, No. 3 (2015), pp. 273-311. 
Ultimate Outcome Premise: $S_{n}$ is not something which should be brought about (because it is horrible, undesirable, etc.).

Conclusion: $S_{0}$ should not be brought about.

How does this scheme fit the one for the basic slippery slope argument? Several remarks are in order to address this question. First there are some slight differences of syntax. The basic scheme talks about carrying out actions while the causal scheme talks about bringing about states of affairs. These differences can be reconciled by treating an agent's carrying out an action, at least for the purposes of relating the two schemes, as being comparable to an agent's bringing about a state of affairs. This is a tricky point, because it requires some considerations in the philosophy of action, but there is no space for that here. But it is not the main problem.

The main problem is that the causal scheme explicitly uses the term 'cause' in the causal sequence premise. So whenever we try to define the controversial notion of cause, the causal scheme is restricted to instances in which one step in the sequence causes the next step (Walton, Reed and Macagno, chapter 3). The basic scheme is more general as it uses the language of one action leading to another action, a language that could include causal steps as well as non-causal steps in the sequence. The basic scheme is general enough to encompass both kinds of steps. Hence it seems reasonable to conclude that the causal scheme can be classified as special subspecies of the more general basic scheme.

Another problem is that the causal scheme as stated above, only has three premises, whereas the basic scheme has six premises. What needs to be done to integrate the two schemes is to revise the causal scheme by adding the three additional premises of the kinds required by the basic scheme. So what needs to be done is to revise the causal scheme of 1992 by adding these three premises to it. By this means the Walton 1992 version causal scheme can be shown to be a subscheme of the basic slippery slope scheme. Basically the same kind of procedure of revision can be used to accommodate the scheme for the precedent slippery slope argument from (Walton, 1992, 155) and the scheme for the full slippery slope argument (Walton, 1992, 199). This makes these earlier versions of these schemes more complex, but it also brings out essential elements of the slippery slope argument shown to be important in this paper.

It is important to realize that the basic slippery slope argument is a species of goal-based reasoning, so-called practical 
reasoning, of which the conclusion can be not only a single action but also a proposal or policy for action. The practical reasoning form of argument applies not only to actions by a single agent but also to multi-agent deliberation in which a group of agents is collectively deciding on what to do in a given set of circumstances. Both practical reasoning and the slippery slope argument are based not only on goals but also on values that can support or detract from such goals. Case in point is the designer babies example from section 5. This argument was directed against the practical wisdom of the decision made by British MPs in 2015 to allow the creation of children with genetic material from three people. The complaint made in the slippery slope argument was that the decision made to approve this procedure by agreeing that it should become policy is the first step on the road to designer babies.

Argument from precedent is a form of argument that has its own scheme (Walton, Reed and Macagno, 2008, 344), and it is this scheme combined with the basic scheme that produces the precedent slippery slope argument. This latter structure has been built on a series of cases in the argumentation scheme for the precedent slippery slope argument in (Walton, 1992, 155). According to this form of argument allowing an exception to a general rule such as a law would lead to a sequence of other precedents, cases that are similar, and that would ultimately lead to an intolerable outcome. The following example from (Walton, 1992, 267-27) was about the burning of an American flag by Gregory Lee Johnson during a political demonstration to protest policies of the Reagan administration. Johnson was convicted of desecration of a venerated object, but the Texas Appeals Court overturned the ruling by arguing that Johnson's act could be classified as "expressive conduct" protected as free speech. The Texas Court of Criminal Appeals argued that Johnson's conduct was a form of "symbolic speech" intended to convey a political message and that, as such, it should be protected by the First Amendment, which includes, under the heading of "speech," such acts as organized demonstrations and the distribution of literature. In the opinion written by Justice William J. Brennan, Jr. in the case of Texas v. Johnson (1989 WL 65231(U.S.), 57 U.S.L.W. 4770), it was argued that Johnson's action can be classified as the expression of an idea, of a kind protected by the First Amendment, quoted from (Walton, 1992, 269-270):

(C) Douglas Walton. Informal Logic, Vol. 35, No. 3 (2015), pp. 273-311. 
We perceive no basis on which to hold that the principle underlying our decision in Schacht does not apply to this case. To conclude that the Government may permit designated symbols to be used to communicate only a limited set of messages would be to enter territory having no discernible or defensible boundaries. Could the Government, on this theory, prohibit the burning of state flags? Of copies of the Presidential seal? Of the Constitution? In evaluating these choices under the First Amendment, how would we decide which symbols were sufficiently special to warrant this unique status? To do so, we would be forced to consult our own political preferences, and impose them on the citizenry, in the very way that the First Amendment forbids us to do.

In (Walton, 1992, 270) this argument was classified as an instance of the precedent type of slippery slope argument. It was classified as a variant of the precedent slippery slope argument where a first step is said to lead to a series of related decisions about what to include. Justice Brennan argued that these subsequent decisions, as one precedent led to another, would culminate in a final outcome where the courts would be imposing their own political preferences on all citizens, a violation of the First Amendment. This outcome would appear to be catastrophic enough in a free country to make the argument against it an instance of the precedent slippery slope argument. In this case there is a sequence of steps in which a legal decision based on precedent is made at each step, and the sequence as a whole leads to a catastrophic outcome.

Any decision made by the Supreme Court is a precedent that takes priority over rulings of other courts, such as a state court, and is binding on them. In the common law system, laws are based on statutes passed by a legislative assembly, but statutory interpretation is based on arguments put forward and decided by the courts. Importantly, whether a law is held to be binding or not in a given case depends on precedents set in similar prior cases. One precedent leads to another as the same rule that held up in one case is applied to another. This chain of argumentation naturally falls into a sequence so that when one case is a precedent for another, the second one can become a precedent for third one, and so forth. An influential ruling by the Supreme Court can easily set such a chain of sequential argumentation into motion.

In this case the sequence of argumentation is impelled forward by four drivers. The first one is its setting in the common 
law system where decisions made on the basis argument from precedent can be binding on other cases. The notion of a precedent and the way it works in the common law system means that a decision can set up a slippery slope argument to attack the prior argument that an action should be taken to make a ruling that leads to the formulation of a new law or acceptance of a new exception should be applied to a given case at issue. A precedent drives an agent forward to the catastrophic outcome by a route that is not causal, but logical. The second driver is that of commitment. The proponent of the argument attacked by the slippery slope argument must concede that if she accepts that the defendant should be convicted of a crime in the present case she must also concede, on pain of inconsistency, that another person in a similar situation should also be convicted of the same crime. The second driver in such a case is the commitment set in place that makes an arguer logically bound to accept a similar proposition even though it is different in some respects from the one it is being compared to. The third driver is the openness or socalled open texture of terms and contexts used in legal argumentation, such as "free speech," that are continually subject to reinterpretation as new cases are decided in the courts. This linguistic factor pertains to the vagueness, or open texture as it is often called, of key legal terms. The fourth driver is the notion of similarity that drives chains of argumentation based on interpretation forward in courts on the principle of treating similar cases alike. This is to assume that argument from precedent is based on argument from analogy.

The argumentation scheme for the precedent slippery slope argument given in (Walton 1992, 155) begins with a premise that the particular case at issue is claimed to be an exception to a rule. The next premise, called the related cases premise, holds that the case at issue is similar to another case, which in turn is similar to a sequence of other cases, so that giving a ruling on the original case, a chain of argumentation will be set up by case to case similarity (argument from analogy). The third premise says that treating the case at issue as an exception to a rule would be intolerable because it would move this chain of argumentation forward so that it would ultimately lead to a catastrophic or intolerable outcome. The conclusion of the argument is that the original case cannot be judged to be an exception to the rule. This form of argument fits into the basic scheme because it has all the same characteristics as those matching the premises and conclusion as those specified in the basic scheme. 
The difference is in the four drivers that can propel a ruling forward from case to case.

\section{What makes the slope slippery?}

The answer to the question of what makes the slippery slope argument slippery begins with the observation (Schauer, 1985, 378 ) that as one proceeds along a sequence of small steps, it is impossible to know where to draw the line. One enters a gray zone, but because of the indeterminacy of this gray zone, there is no specific point along the sequence where it begins (Walton, 1992, 50-52). But at some undetermined point within this gray zone, one has lost control, meaning that there is no longer the possibility of turning back (Walton, 1974). After reaching that point one is impelled down the remaining part of the slope to the catastrophic outcome at the bottom.

From the drug example one can see the importance of the possibility that there can be two or more drivers pushing the sequence of argumentation forward into the gray zone, through it and beyond until the agent is on the steepening part of the slope where it is impelled downward with no control toward the catastrophic outcome. The gray zone (gray area) within which control is lost is represented in figure 2 .

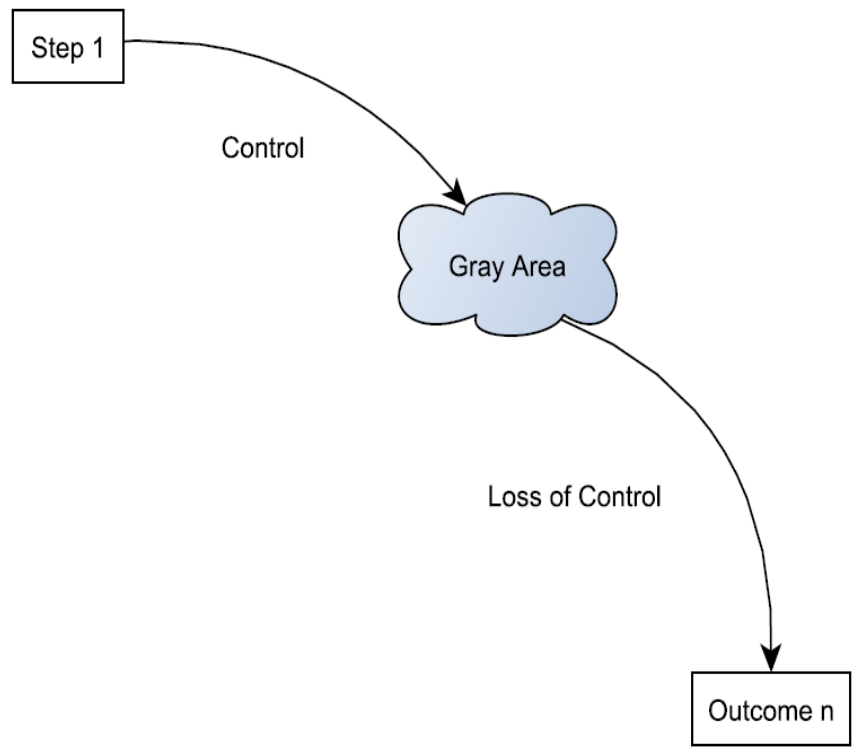

Figure 2: Slippery Descent of the Slippery Slope Argument

(C) Douglas Walton. Informal Logic, Vol. 35, No. 3 (2015), pp. 273-311. 
On the part of the descent before reaching the gray zone and entering into it, the agent's actions are impelled forward by the pleasure of taking the drug. At some undefined point in the gray zone the agent has become addicted to the drug and now it is much more difficult to stop taking it, for some agents perhaps even impossible, because of the increasingly painful withdrawal symptoms. The paradox of the heap (bald man) is not itself a slippery slope argument, but its feature of the gray area is the key component that explains what makes the slippery slope slippery. What makes it slippery, as shown precisely in the scheme proposed in section 7 , is the connection between the gray area and the loss of control once one has entered into it.

Now we are in a position to obtain a much clearer grasp of how the slippery slope argument, even though it is a species of argument from negative consequences, is distinct as a special form of argument in its own right with identifiable characteristics that mark it out. The first characteristic is that the transition from the action to its consequence must take place over an intervening sequence of actions $A_{0}, A_{1}, A_{2}, \ldots, A_{x}, \ldots A_{y}, \ldots, A_{n}$ such that each action in the sequence leads to the next one, even though the individual links between pairs of actions can be quite different. The second characteristic is that the sequence contains a subsequence $A_{x}, \ldots A_{y}$ called the gray zone where $x$ and $y$ are indeterminate points. The third characteristic is that once the sequence of actions enters the gray zone (area) the agent loses control over whether to continue taking the actions in the sequence or not. The fourth characteristic is that once the sequence of actions proceeds out of the gray zone, the agent has lost control, and is impelled if not inevitably drawn much more strongly, to the end point of the sequence, the catastrophic outcome. These four characteristics combine so that once the agent has taken the first step $A_{0}$, the slippery slope sequence will kick into place and generate a movement of its own that will carry the agent forward more and more strongly toward the catastrophic outcome, causing the agent to lose control in an insidious manner.

The slippery slope argument does essentially involve vagueness on a continuum, but that is far from the whole story of it. A vitally important component is the loss of control as the sequence of actions is moved forward by the drivers over the gray area toward the ultimate outcome. Once the structure of the slippery slope argument has been precisely modeled, and a reasonably clear case, namely the drug example, is given to show how such an argument can be reasonable in some instances, the force 
and legitimacy of the argument can be appreciated. The essential structure of the slippery slope argument is shown in figure 3.

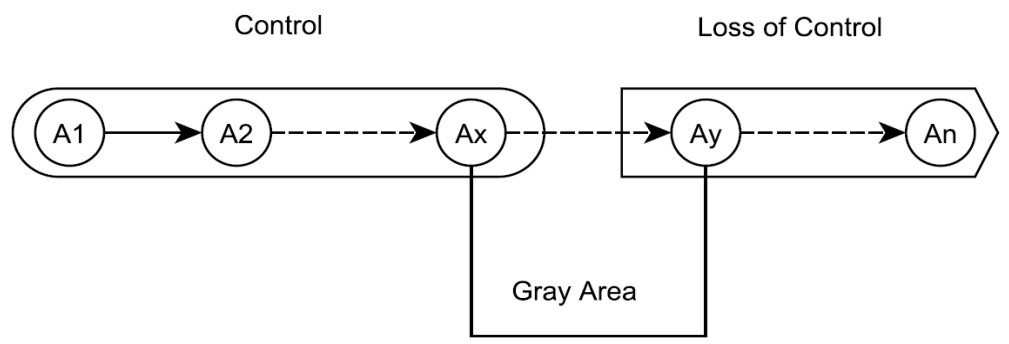

Figure 3: The Sequence and the Gray Area

The essential structure of the argument is the sequence of actions beginning with the first action $A_{l}$, the moving forward of the sequence through the gray area, loss of control at some point as the sequence goes through the gray area, and the ultimate descent to the catastrophic outcome $A_{n}$. What makes the slippery slope slippery is the indeterminacy of the gray area, which means that the point at which the agent loses control cannot be anticipated in advance. The agent can rationalize, thinking that it can stop at any point, and this is true at first, but insidiously becomes false in a way that is impossible to anticipate, and so the agent becomes trapped. Once the slippery slope argument is explained in this way, it is easy to appreciate why it is a scary argument, and one that a rational agent should pay careful attention to. On the other hand, because it is such a scary argument, it is also easy to take advantage of it to try to manipulate an audience with sleight-of-hand. There are always two sides to it. It can be attacked as well as defended, and needs to be evaluated on a balance of considerations where the pro and con arguments are displayed together in an argumentation graph.

We can briefly illustrate how this procedure works by looking back to the example of the designer babies slippery slope argument from section 5. In figure 4, the action of approving the procedure of genetic intervention is shown in the rectangle containing "Approve Procedure." The pro-argument supporting this conclusion is labeled in the circle containing the notation $+\mathrm{a} 1$. Below this argument there is a second argument, a con argument labeled -a2, attacking the pro-argument $+\mathrm{a} 1$. Finally there is a con argument -a3 attacking the argument $-\mathrm{a} 2$. The con argument is based on the premise stating that approving one procedure does not mean automatically approving others. In the figure, this premise is indicated by the notation "Not Automatically." 


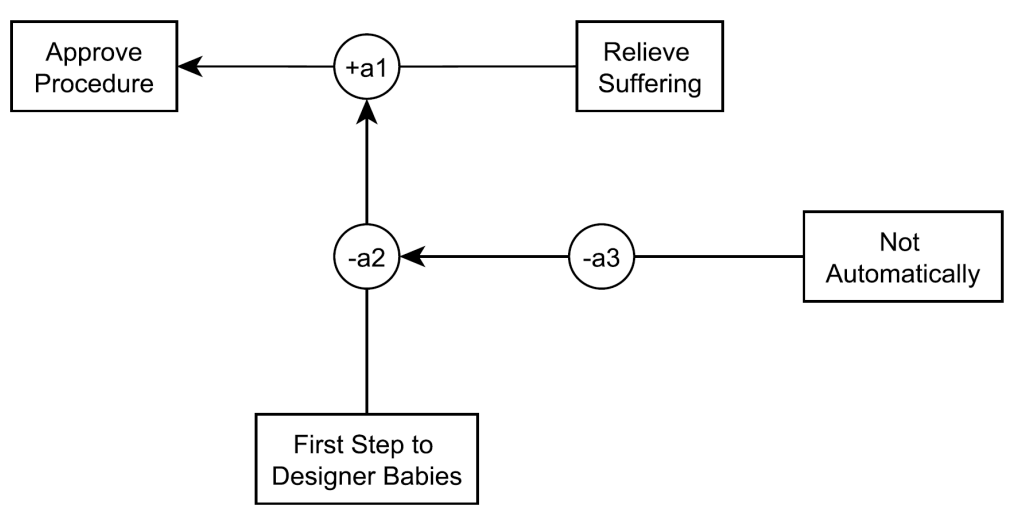

\section{Figure 4: Arguments in the Designer Babies Example}

The conclusion of argument a1, the action of approving the procedure for transferring the nucleus of an egg cell with damaged mitochondria into the body of an egg that is not damaged, is shown in the rectangle at the top left of figure 4 . The slippery slope argument is shown in the figure as this argument a2. The counterargument to the slippery slope argument is the argument a3, which has the premise that approving one procedure does not mean automatically approving others. So argument a1 is a proargument supporting the ultimate conclusion. Argument a2 is a con argument, a slippery slope argument attacking argument a1. And argument a3 attacks argument a2. It is an instance of two undercutters, where an undercutter is defined following Pollock (1995) as an argument attacking the inferential link between the premises and conclusion of a prior argument. This example is a case of one undercutter undercutting another undercutter.

This argument, as shown in figure 4, is a classical example of the formalism widely used in artificial intelligence called abstract argumentation frameworks. In this formal model, arguments can take on one of three values: accepted (in), rejected (out), or neither accepted nor rejected. In this formalism, an argument is accepted (in) as long as it is not attacked by any other argument that is accepted (in). But an argument is rejected (out) if it is attacked by any other argument that is accepted (in). If the first argument is attacked by a second argument that is accepted (in) then the first argument is refuted (out). But if the second argument is attacked by a third argument that is accepted (in) in the second argument becomes unacceptable (out). Therefore, the status of the first argument is automatically restored. It is changed from unacceptable (out) to acceptable (in). Neverthe-

(C) Douglas Walton. Informal Logic, Vol. 35, No. 3 (2015), pp. 273-311. 
less while this formalism does model some main aspects of the slippery slope argument used in the designer babies example, it can be improved significantly by adding the argumentation scheme for the basic slippery slope argument into the structure of the figure and taking this scheme into account.

Above it was noted that the slippery slope argument is shown in figure 4 as argument a2. In some formal argumentation systems of the kind developed in artificial intelligence, such as ASPIC+ (Prakken 2010) and CAS (Gordon 2010). In CAS, for example, the argumentation schemes, including the one for the slippery slope argument that is contained in the computational structure CAS, fit into the circular argument nodes. In this instance, the argument node containing the notation a 2 is taken to be an instance of the slippery slope argument. This means that the requirements of the scheme need to be built into the argument displayed at node a2. In such systems, a slippery slope argument is modeled in such a way that it does not successfully refute the original argument it was directed against unless the refuting argument fits the requirements of the scheme, in this instance the basic scheme for the slippery slope argument. This means that all the premises of the slippery slope argument shown in the basic scheme have to fit the example, and the slope argument is not adequate to refute the argument it was directed against unless it can be shown that all the premises of the basic scheme are present in the text, or if they are implicit they can be made explicit using evidence from the text, in this case the argument as it was put forward in the Economist article. In this instance the argument that this is the first step to designer babies is meant to be a slippery slope argument, and is only possible if taken this way, but nevertheless states only one of the premises of the basic slippery slope scheme. No evidence indicating or supporting the other premises is provided. Hence the slippery argument fails in the designer babies example, judging by the text given as the example.

The fundamental problem is that the model of the argumentation shown in figure 4 only has the first step and the conclusion of the basic argumentation scheme for the slippery slope argument. None of the other premises has been explicitly stated. But the argument cannot be evaluated as a slippery slope argument, using the basic scheme to represent the logical form of that type of argument, until the other implicit premises have been made explicit. Until this has been done, if the argument a 2 in the designer babies is supposed to be a slippery slope argument, the counterargument a3 is basically correct to make the criticism 
that this prior argument is weak, because it does not show how one step in the sequence automatically goes into a gray area leading to loss of control, and ultimately to the allegedly catastrophic conclusion of approving a procedure that will produce "designer babies."

The example shows how an argumentation scheme can be used within a formal argumentation model as a device for argument evaluation. An argument can be shown to be weak if it omits or fails to substantiate some of the premises required by the scheme. A more general discussion on how formal argumentation are applicable to such cases is offered in section 10.

\section{Critically questioning a slippery slope argument}

The argumentation scheme for the slippery slope argument presented in (Walton 1992) has a list of critical questions attaching to this scheme. Slippery slope arguments are defeasible, meaning that they only hold tentatively, and may be defeated by the asking of critical questions or the putting forward of counter arguments. Once such an argument has been identified, the next question is how to respond to it appropriately by examining its pros and cons.

There are basically three ways to attack an argument in formal argumentation systems such as ASPIC+ and CAS: you can attack its premises, you can attack the inferential link between premises and conclusion, or you can attack the conclusion directly by posing a counterargument against it, making the claim that the conclusion is false, or not acceptable based on the evidence. However, there are also other ways to attack an argument that someone puts forward as a slippery slope argument. As shown by the examples above, such an argument may not really be a slippery slope argument, but maybe better fit some other argumentation scheme such as argument from negative consequences, without fitting the requirements necessary to make it a genuine slippery slope argument. To attack the argument in this way, the critic needs to bring forward the argumentation scheme for the slippery slope argument, and also perhaps explain the necessary characteristics that identify an argument as fitting the slippery slope category, and show that the given argument fails to meet one or more of these requirements. This move shifts the burden of proof onto the proponent of the argument to bring forward more evidence to show that it fits the slippery slope category. Failure to meet these requirements means that the argu-

(C) Douglas Walton. Informal Logic, Vol. 35, No. 3 (2015), pp. 273-311. 
ment cannot be criticized as a fallacious slippery slope argument.

Another way to attack the slippery slope argument is to ask critical questions matching the argumentation scheme (Walton, 1992). These critical questions represent standard ways of finding gaps in the argument and probing into its weak points. They are therefore helpful to the beginner who has not been trained in argumentation and may find it difficult to respond to a cleverly expressed slippery slope argument, even though the argument is in such a compressed form that is full of all kinds of holes. One of the most important critical questions is whether there is a bright line that can be placed into the gray zone to stop the slippery slope from moving forward. This form of attack was in fact the move made in the designer babies example.

What makes a slippery slope slippery is this gray zone, and what makes the gray zone possible is indeterminacy of a kind that offers no clear and precise point demarcating the action at which the agent will lose control, and afterwards descend inevitably to the catastrophic outcome. However, legal argumentation is full of cases where laws are enacted by legislatures and then interpreted by court rulings that tighten up the open texture of terms that are difficult to precisely define. In some cases these proposed criteria do not work very well, for example because they lead to a law that is unenforceable. But in other cases the proposed criteria work very well, especially when they are tested and refined by new cases that challenge them. The procedure is one of case-based reasoning, where laws are applied to cases using precedents from previous cases. In other words, law already has a working system in place for dealing with slippery slope arguments, or even preventing them from arising. Law also illustrates the possibility of finding a bright line by devising a legal rule that can be used to cut through the gray zone preventing the slippery slope from descending to the catastrophic outcome.

The following objection seems to pose a problem. A slippery slope critique seems to express the message that the critic is advising the proponent not to take the initial step, for even by not taking the initial step she might already lose control. It would seem that if not, the proponent could easily answer: "Thanks for the warning, but I will stop before I enter the gray area." This possibility seems to pose in connection to the basic argumentation scheme as a model of the logical form of slippery slope arguments, because it seems that the initial action must already

(C) Douglas Walton. Informal Logic, Vol. 35, No. 3 (2015), pp. 273-311. 
reside within the gray zone, contravening the requirements of the basic scheme.

The reply to the objection is that as the slippery slope argument is stereotypically used, it is not meant to advise the proponent not to take the initial step for the reason that even at this initial step she might already lose control. That is an additional argument that might optionally be added on in some cases. The critic might add, in a case about considering trying heroin for example: "Some people can resist taking heroin even after trying it several times, so it is possible that you could stop before entering the gray area, but there are also some rare instances where once a person has taking this drug even once, the impact is so pleasurable that she cannot resist trying it again." However, this additional argument should not be seen as part of the slippery slope argument. It is an add-on that might make the argument even powerfully persuasive to some respondents. But also runs the risk of seeming unpersuasive to others. For example adolescents can be very suspicious that warnings about taking drugs are exaggerated and used as scare tactics.

There is also another objection to be considered. The nature of slippery slope arguments being what they are, they are arguments about what might or will happen in the distant future. A critic might say therefore, that it is impossible to prove or disprove them. But this is too harsh a stance. We use arguments that depend on predictions about the future all the time. Argumentation from negative consequences is one of the most common forms of argument in human deliberations, and it is an argument about what can or will happen in future. The problem with slippery slope arguments is that they generally are about what will happen in the distant future if you carry out a particular action now that might lead to a lengthy sequence of actions resulting in some highly unpleasant outcome over the long term at some endpoint. Of course a critic can try to respond to such an argument by saying that it's all speculation. But if the audience is worried enough about the catastrophic outcome, and if the audience is inclined to accept the premises of the slippery slope argument, even if they have not been explicitly stated or supported by the proponent, this type of argument can be powerfully persuasive. Indeed, we have argued here that it can be in principle a reasonable argument in some instances, if supported well enough by the evidence that is available, even though it requires extrapolating the argument quite a distance into the future.

(C) Douglas Walton. Informal Logic, Vol. 35, No. 3 (2015), pp. 273-311. 
For these reasons, a line of counterattack that is very important for a critic to work on is to argue that a bright line criterion can be furnished that will stop the slippery slope by dissipating the gray zone. This line of attack can be put forward even if the critic does not know at the present time how that bright line will be defined precisely, or even what it is. For as argued above, it could be quite possible that the legal system can do away with the slippery slope by means of its usual methods of case-based reasoning, and testing criteria in trials.

\section{Conclusions and further research tasks}

In this paper, it has been shown that slippery slope arguments are in principle reasonable arguments, or can be, because they are a species of arguments from negative consequences. These findings support the conclusion drawn by Govier (1982, 316) that a slippery slope argument may make many mistakes, but need not make any. Slippery slope arguments can be reasonable in some cases of intelligent deliberation, as shown by the example of advice-giving deliberation in which the father advises his son that taking drugs would not be a good idea because it can lead to loss of control resulting in a catastrophic outcome. A reasonable slippery slope argument of this kind has four basic characteristics that can be summed up as follows. First, there must be a framework of deliberation in which one agent is advising another on a choice of action. Second, there must be a sequence of actions leading from the action to be chosen to an ultimate outcome. Third, there must be a gray area in the middle region of the sequence in which the agent will lose control. Fourth, once the agent has lost control, he or she will be impelled along the sequence leading to an ultimate catastrophic outcome.

It has also been easy to assume that the slippery slope argument is fallacious for three reasons: (1) because it is a long-term argument that goes far into the future and necessitates long-term planning, (2) because it is such a complex form of argument that it is hard to grasp its structure, and (3) because it is often associated with arguments on euthanasia and eugenics and the like, arguments that seem exaggerated because they make emotional appeals and use emotional terms such as "euthanasia" and "eugenics" associated with mass murder programs of the Nazi period in Germany (Bashford and Levine, 2010).

(C) Douglas Walton. Informal Logic, Vol. 35, No. 3 (2015), pp. 273-311. 
Because slippery slope arguments typically go so far into the future, and have so many additional components than the more common kinds of arguments from negative consequences, they are hard to support, but they need much support, often empirical support deriving from the circumstances of each individual case. To be a slippery slope argument, an argument has to fit the requirements of having the right premises and conclusion to fit the argumentation scheme for that type of argument. The argumentation scheme proposed in this paper helps with this job by carrying through a series of steps in the procedure for the identification method. Allied with better knowledge of how the basic slope argument is related to its subtypes, such as the linguistic, causal and precedent types, the basic scheme is a tool useful for analyzing and evaluating slippery slope arguments.

This paper goes some way toward casting doubt on the thesis of Lode $(1999,1492)$ that there is no single distinctive form of the slippery slope argument. His argument is based on the premise that different slippery slope arguments hold that stepping on the slope is objectionable for different reasons. The conclusion he has drawn is that slippery slope arguments are a family of related arguments rather than a class of arguments whose members all share the same form. To conclusively refute this thesis we would need to analyze a large and representative number of examples of slippery slopes, including many from law, and show that all of them can be shown to be instances of the basic slippery slope argumentation scheme. This task can be proposed as a topic for further research in which the hypothesis to be tested is the scheme for the basic slippery slope proposed in this paper. The procedure required to apply this scheme to examples that purport to be genuine instances of slippery slope arguments has been built in this paper.

As applied to instances of the slippery slope argument, the procedure has the following steps. First of all, frame the argument in a context of deliberation in which one agent is giving advice to another. One of the agents is contemplating taking some particular step, and the other is advising the first agent not to take that step because it will lead forward by a sequence of actions that will, during a gray zone, run out of control leading to an outcome that the second agent evaluates as highly negative, catastrophic or intolerable. Once the argument has been situated in this context of a deliberation, the next step is to see if it has all of the characteristics of the slippery slope argument. For these purpose the application of the basic argumentation scheme for the slippery slope type of argument to the textual 
data of the given argument is required. Some arguments that are said to be slippery slopes and that look like a slippery slope may not fit all of the requirements. In particular, it is necessary to analyze the given argument to find the three key components of it, the gray zone, the sequence and the drivers.

For length reasons, naming and classification issues were not extensively treated in this paper. Various other names for the slippery slope argument, such as the thin edge of the wedge argument, the dam burst argument, the camel's nose in the tent argument, and so forth, are present in the literature. No serious attempt has been made to confront the issue of whether these terms are equivalent to the slippery slope name, or whether they represent different types of arguments related to the slippery slope argument, such as species of slippery slope argument. This subject can also be further investigated using the basic scheme.

The contextual variability of use of the slippery slope argument, and related types of arguments it is built on, such as arguments from goal-directed practical reasoning and arguments from negative consequences is a high level problem that cannot be solved in this paper. But it is an important problem, and some analysis of how it impacts on the analysis of the slippery slope argument put forward in this paper are useful. Those writing on biomedical issues concerning the slippery slope argument tend to see the framework of its use as a deliberation dialogue in which one party is considering carrying out a particular action, such as a genetic intervention, and the other party in the dialogue is using a slippery slope argument to suggest that carrying out this section would be the first step in the sequence of following events that might lead to a disastrous outcome. Commentators treated above such as Corner et al., Saliger, and Holtug, fall into this category. However, as the six basic characteristics of the slippery slope argument proposed in (Walton, 1992, 208) make clear, this analysis is much broader, because it includes cases of slippery slope arguments used in other types of dialogue, such as persuasion dialogue and negotiation dialogue. This analysis is based on a dialectical framework where the steps in the slippery slope argument are modeled through the device of a commitment store in a dialogue. The commitment store is a database of propositions such that at each step of the slippery slope argument, as it unfolds in the dialogue between the two sides, propositions can be inserted into the database or deleted from it, depending on the dialogue protocol and the kinds of speech acts put forward by each party unity to move.

(C) Douglas Walton. Informal Logic, Vol. 35, No. 3 (2015), pp. 273-311. 
Each instance of an argument in a natural language text is different and needs to be treated as an individual case, but formal models are sometimes very useful tools to set out normative requirements for what should count as a correct argument of a particular type. Argumentation schemes need to be part of such more formal models, and many tools commonly used in argumentation and informal logic such as argument diagrams have a formal structure. Informal logic is a field of study that grew from the perception that the formal methods used during the 20 th century, especially classical deductive logic, were not as useful as one might like to study real examples of argumentation in natural language. Formal argumentation methods developed in computer science took this challenge seriously, and responded by offering more flexible formal systems and tools designed to be helpful for the tasks of informal logic. These tasks were taken to include the identification, analysis and evaluation of arguments, and even the task of argument invention. The outcome of these developments is that argumentation theory has grown into an interdisciplinary area that integrates and applies tools and methods from different fields. This interdisciplinary aspect of argumentation was made clearly apparent in a recent invitation from the director of the Leibniz Center for Informatics to the author to participate in a one-week Schloss Dagstuhl ${ }^{3}$ seminar in Germany entitled Natural Language Argumentation: Mining, Processing, and Reasoning over Textual Arguments. The statement was made in the invitation that because argumentation is an inherently cross-disciplinary topic involving philosophy, communication studies linguistics, and computer science, where different interpretations, analyses, and uses of arguments are proposed and applied, there needs to be progress not only within each domain, but also in bridging these various disciplines.

The literature on formal argumentation systems has now been expanded to include formal multiagent deliberation dialogue systems based on practical reasoning (McBurney, Hitchcock and Parsons, 2007; Kok et al., 2011; Medellin-Gasque, et al., 2011), a form of argument closely related to slippery slope arguments. It is important for new research on such arguments to take such formal argumentation systems currently being developed in multiagent systems research and artificial intelligence into account. Now that formal argumentation models of dialogue are available in which slippery slope arguments and related arguments such as

\footnotetext{
${ }^{3}$ Dagstuhl supports computer science by organizing advanced seminars on current topics and problems in informatics that bring together personally invited scientists from academia and industry all over the world.
}

(C) Douglas Walton. Informal Logic, Vol. 35, No. 3 (2015), pp. 273-311. 
argument from negative consequences can be modeled, researchers studying informal logic should take advantage of the tools they provide.

The primary type of dialogue in which slippery slope arguments are used, for example in biomedical issues about genetic therapy, is deliberation dialogue. In this type of dialogue one agent, the proponent, is faced with a decision about what to do in a particular set of circumstances, and the other agent, the respondent, is playing the role of critic by asking critical questions and putting forward counterarguments directed to the action the proponent has tentatively chosen to go forward with (McBurney, Hitchcock and Parsons, 2007; Kok et al., 2011; MedellinGasque, et al., 2011).). This type of dialectical situation is the prototypical case in which forms of argument such as argument from negative consequences and the slippery slope argument are primarily used. The contribution of this paper is to provide a basic argumentation scheme for the slippery slope argument that can be fitted into the broader framework of the analysis of slippery slope arguments given in (Walton, 1992) that is commitment-based, and that covers uses of slippery slope arguments in other types of dialogue as well as that of deliberation.

Further research is also needed to show more precisely how the slippery slope scheme is related to the scheme for practical reasoning, a scheme that is very closely related to the scheme for argument from negative consequences. Especially important in this regard is the scheme for value-based practical reasoning. In an argument from negative consequences, the consequences are described as "bad" or "negative," meaning that they are low in a preference ordering exhibiting a scale of values representing the values applicable in a particular case. Similarly, in a slippery slope argument, the ultimate outcome is described as a catastrophe, implying that it has to be very low on the preference ordering, so low that it is imperative to take steps to avoid it.

How these factors work in to the analysis of the slippery slope argument is a subject of argumentation research that needs to be investigated in more detail. Although slippery slope arguments are complex, because they are made up of a network of simpler arguments nested together into the one large argument, the best way to grasp their complexity and help us to understand them is to classify this cluster of arguments so that it can be shown how the slippery slope scheme is built up from other schemes, such as argument from negative consequences. Although the basis for this work is built in the present paper, continuing it requires a 
research on classification of argumentation schemes covering all the known schemes.

Another main problem arising from the paper was that of dealing with compressed slippery slope arguments, arguments that are claimed to be slippery slope arguments that are expressed in such a compressed form that many of the necessary ingredients for this type of argument are not explicitly stated. What needs to be done in such cases is to use the basic argumentation schemes to search the text of discourse in a given case for the missing elements. This procedure is a typical argumentation task associated with enthymemes, arguments with missing premises or a missing conclusion. It can be used to help evaluate arguments, such as the slippery slope argument, using formal argumentation systems currently being developed in AI, such as ASPIC+ and CAS. Dealing with these problems is beyond the scope of this paper, but enough of the structure of the slippery slope argument has now been identified to provide a basis to help this research work move forward.

\section{Acknowledgements}

The research for this paper was funded by Insight Grant 4352012-0104 from the Social Science and Humanities Research Council of Canada.

\section{References}

Bashford, A. and Levine, P. (2010). Introduction: Eugenics and the Modern World. The Oxford Handbook of the History of Eugenics, A. Bashford and P. Levine (Eds.), pp. 3-24. Oxford: Oxford University Press.

Beardsley, M. C. (1966). Thinking Straight, $1^{\text {st }}$ ed. Englewood Cliffs, NJ: Prentice-Hall.

Beardsley, M. C. (1975). Thinking Straight, $4^{\text {th }}$ ed. Englewood Cliffs, NJ: Prentice-Hall.

Burgess, J. A. (1993). The Great Slippery Slope Argument, Journal of Medical Ethics 19: 169-174.

Cicero, M. T. (1951). Academica, Trans. H. Rackham. Loeb Library Series. Cambridge, Mass: Harvard University Press.

Corner, A., Hahn, U. and Oaksford, M. (2011). The Psychological Mechanism of the Slippery Slope Argument, Journal of Memory and Language 6: 133-152.

(C) Douglas Walton. Informal Logic, Vol. 35, No. 3 (2015), pp. 273-311. 
Damer, T. E. (1980). Attacking Faulty Reasoning. Belmont, CA: Wadsworth.

Gordon, T. F. (2010). The Carneades Argumentation Support System, Dialectics, Dialogue and Argumentation, ed. C. Reed and C. W. Tindale, London: College Publications.

Govier, T. (1982). What's Wrong with Slippery Slope Arguments? Canadian Journal of Philosophy 12: 303-316.

Grice, H. P. (1975). Logic and Conversation, The Logic of Grammar, eds. D. Davidson and G. Harman. Encino, California: Dickenson, 64-75.

Holtug, N. (1993). Human Gene Therapy: Down the Slippery Slope, Bioethics 7(5): 402-419.

Kneale, W. and Kneale, M. (1962). The Development of Logic. Oxford: Oxford University Press.

Kok, E. M., Meyer, J. J. C., Prakken, H., and Vreeswijk, G. A. W. (2011). A Formal Argumentation Framework for Deliberation Dialogues. In Argumentation in Multi-Agent Systems, volume 6614 of Lecture Notes in Computer Science. Springer Berlin Heidelberg.

Launis, V. (2002). Human Gene Therapy and the Slippery Slope Argument, Medicine, Health Care and Philosophy 5(2): 169179.

Lode, E. (1999). Slippery Slope Arguments and Legal Reasoning, California Law Review 87(6): 1469-1544.

Macagno, F. and Walton, D. (2013). Implicatures as Forms of Argument, Perspectives on Pragmatics and Philosophy, A. Capone, et al. (Eds.), pp. 203-224. Berlin: Springer.

McBurney, P., Hitchcock, D., and Parsons, S. (2007). The Eightfold Way of Deliberation Dialogue. International Journal of Intelligent Systems 22(1): 95-132.

Medellin-Gasque, R., Atkinson, K., McBurney, P., and BenchCapon, T. (2011). Arguments over Cooperative Llans. In Theory and Applications of Formal Argumentation, volume 7132 of Lecture Notes in Computer Science. Berlin: Springer.

Pollock, J. L. (1995). Cognitive Carpentry. Cambridge, MA: The MIT Press.

Prakken, H. (2010). An Abstract Framework for Argumentation with Structured Arguments. Argument and Computation 1: 93-124.

Rizzo M. J. and Whitman, D. C. (2003). The Camel's Nose in the Tent: Rules, Theories and Slippery Slopes, UCLA Law Review 51: 539-592. 
Saliger, F. (2007). The Dam Burst and Slippery Slope Argument in Medical Law and Medical Ethics, Zeitschrift fur Internationale Strafrechtsdogmatik 9: 341-352.

Schauer, F. (1985). Slippery Slopes, Harvard Law Review, 99(2): 361-383.

Sidgwick, A. (1910). The Application of Logic. London: MacMillan and Co.

Thouless, R. H. (1930). Straight and Crooked Thinking. London: English Universities Press.

Van der Burg W. (1991). The Slippery Slope Argument, Ethics 102: $42-65$.

Volokh, E. (2002). The Mechanisms of the Slippery Slope, Harvard Law Review, 116(4), 1026-1137.

Walton, D. (1974). Control. Behaviorism, 2(2): 162-171.

Walton, D. (1992). Slippery Slope Arguments. Oxford: Oxford University Press.

Walton, D. (1996). The Argument of the Beard, Informal Logic 18(2\&3): 235-259.

Walton, D., Reed, C. and Macagno, F. (2008). Argumentation Schemes. Cambridge: Cambridge University Press. 